\title{
PREFERENCE RELATIONS AND MEASURES IN THE CONTEXT OF FAIR DIVISION
}

\author{
JULIUS B. BARBANEL AND ALAN D. TAYLOR
}

(Communicated by Andreas R. Blass)

\begin{abstract}
One of the most well-known metaphors in the mathematical theory of fair division concerns the problem of dividing a cake among $n$ people in such a way that each person is satisfied with the piece he or she receives, even though different people value different parts of the cake differently. Our concern here is with how an individual's preferences are formalized. David Gale has pointed out that although most of the deeper results in the field assume that preferences are given by an additive measure, the fundamental algorithms in the field require only that preferences be given by a binary relation satisfying a few natural properties. We introduce here one additional condition-an Archimedean property that obviously holds if the relation is induced by a measure-and we show that a preference relation satisfying Gale's conditions is induced by a finitely additive measure if and only if it satisfies this Archimedean property.
\end{abstract}

\section{INTRODUCTION}

Suppose $\mathscr{A}$ is an algebra of subsets of the set $C$. A preference relation $(R)$ on $\mathscr{A}$ is a binary relation, defined for pairs of sets from $\mathscr{A}$, that is reflexive, transitive, and complete (on $\mathscr{A}$ ). If $R$ is such a relation, then the corresponding relations of indifference $(I)$ and strict preference $(P)$ are given by

(i) $X I Y$ iff $X R Y$ and $Y R X$, and

(ii) $X P Y$ iff $X R Y$ and $X Y Y$.

It is easy to see that the indifference relation $I$ and the strict preference relation $P$ must also be transitive. The term "indifferent" is suggestive when one thinks of the preference relation as reflecting the tastes of an individual, but even here it really corresponds to a property of the person and not to a property of the pair of sets. In order to avoid speaking of two sets as being "indifferent", we will often use the term equivalent in place of indifferent.

The context in which we are interested arises from the well-known problem of fair division that is usually phrased in terms of a cake metaphor. That is,

Received by the editors February 27, 1993 and, in revised form, September 3, 1993.

1991 Mathematics Subject Classification. Primary 28A60; Secondary 04A05.

The second author's research was partially supported by National Science Foundation Grant DMS-9101830. 
suppose we have a cake $C$ and $n$ people who value different parts of the cake differently. Is there a way to divide the cake among the $n$ people so that each person is satisfied with the piece he or she receives? Expository treatments of this problem can be found in [DS] and [BT].

Satisfaction may be defined, of course, in several different ways. For example, one might be satisfied only if he or she received at least $1 / n$ of the cake ("proportionality") or only if he or she would not trade that piece for the one received by someone else ("envy-freeness").

The real issue we want to confront here, however, lies with the phrase "who value different parts of the cake differently". Economists have traditionally dealt with the context in which the cake corresponds to a finite collection of divisible homogeneous goods, while mathematicians have dealt with the context in which it corresponds to a single divisible heterogeneous good. We are interested here only in the latter. But even in this context there is the fundamental distinction, explicitly pointed out by Gale [G], between the context in which these "values" are formally represented by a preference relation and the context in which they are represented by a finitely or countably additive measure. Continuing along the lines suggested by Gale, we make

Definition. Suppose $R$ is a preference relation defied on an algebra $\mathscr{A}$ of subsets of $C$. Then $R$ will be called a $C D$ preference relation (" $\mathrm{CD}$ " for "cake-division") if $C$ is strictly preferred to the empty set and the relation $R$ satisfies the following three postulates:

(1) The Partitioning Postulate (PP). If $X \in \mathscr{A}$ and $k$ is a positive number, then $X$ can be partitioned into $k$ pairwise disjoint equivalent sets which are in $\mathscr{A}$.

(2) The Trimming Postulate (TP). If $X$ is preferred to $Y$, then there exists a subset $Z$ of $X$ which is equivalent to $Y$ and in $\mathscr{A}$.

(3) The Weak Additivity Postulate (WA). If $X$ is preferred to $Y, A$ is preferred to $B$, and $X$ and $A$ are disjoint, then $X \cup A$ is preferred to $Y \cup B$. If either of the two preferences in the hypothesis is strict, then so is the preference in the conclusion.

Our definition of a CD preference relation has the "more is better" property built into it in two places: the assertion that $C$ is strictly preferred to the empty set and the requirement in Weak Additivity that $X$ and $A$ be disjoint but not necessarily $Y$ and $B$. If we wished to depart from the cake-cutting tradition, then mathematical naturality would suggest that we state WA with disjointness for both pairs and require only that $C$ not be equivalent to the empty set. One could then define an "upward monotone CD preference relation" as one for which $X \subset Y$ implies $Y R X$ and a "downward monotone CD preference relation" as one for which $X \subset Y$ implies $X R Y$. Both kinds of monotone relations can be obtained by a measure in the obvious way, although it is easy to construct preference relations that satisfy neither kind of monotonicity. For example, if $\{A, B\}$ is a partition of $C$ and $\mu$ is a measure on $C$ giving positive measure to both $A$ and $B$, then we could say $X R Y$ holds precisely when $\mu(X \cap A)-\mu(X \cap B) \geq \mu(Y \cap A)-\mu(Y \cap B)$. It is easy to see that this is an example of what one would call a nonmonotone CD preference relation. Our results directly apply to the upward monotone case and can be adapted easily to the downward monotone case. 
If $R$ is a $\mathrm{CD}$ preference relation, then an equipartition $\mathscr{X}$ of a set $X$ is a partition of $X$ into pairwise equivalent sets. (Here, and in all that follows, we will assume that all sets mentioned belong to some common algebra $\mathscr{A}$ on which all the preference relations under discussion are defined.)

The following notion is central to our considerations.

Definition. Suppose $R$ is a CD preference relation defined on an algebra $\mathscr{A}$ of subsets of $C$. Then a set $X \in \mathscr{A}$ will be called an infinitesimal if $X$ is strictly preferred to the empty set but every set $Y \in \mathscr{X}$ is preferred to $X$ whenever $\mathscr{X}$ is a finite equipartition of $C$ (regardless of the number of sets in $\mathscr{X}$ ).

If $\mu$ is a finitely additive measure on some algebra $\mathscr{A}$ of subsets of $C$, then one can define a preference relation $R$ on $\mathscr{A}$ by setting $X R Y$ iff $\mu(X) \geq \mu(Y)$. If the measure satisfies the obvious analogues of the Partitioning Postulate and Trimming Postulate, then $R$ is a CD preference relation. It is, of course, trivial to see that such a preference relation cannot have infinitesimals. This leads to

Definition. A CD preference relation $R$ defined on the algebra $\mathscr{A}$ of subsets of $C$ will be called an Archimedean $C D$ preference relation if it also satisfies:

(4) The Archimedean Postulate (AP). There are no infinitesimals.

Gale [G] introduced versions of the first three postulates above to underscore the point that, although much of the research in the area of cake division has been in the context of finitely or countably additive measures, the classic algorithms in the area (e.g., those of Steinhaus, Banach-Knaster, and SelfridgeConway-see [BT]) require only (what we are calling) CD preference relations.

Our goal in what follows is to show that, in one sense, this added generality is somewhat illusory, although, in another sense, perhaps quite fundamental. In particular, we prove

Theorem 1. If $R$ is an Archimedean CD preference relation on $\mathscr{A}$, then there exists a finitely additive measure $\mu$ on $\mathscr{A}$ so that $X R Y$ holds precisely when $\mu(X) \geq \mu(Y)$.

Theorem 2. There exists a $C D$ preference $R$ that is not Archimedean (and hence does not arise from any measure).

\section{Proof of Theorem 1}

The basic idea of the proof of Theorem 1 is as follows. The Partitioning Postulate allows us, for any $n$, to split $C$ into $n$ equivalent pieces. If the preference relation is to be induced by a finitely additive measure, then each such piece must have measure $1 / n$, and the union of any $r$ of these pieces must have measure $r / n$. However, if a set $X$ is assigned measure $r / n$ on the basis of one such partition, then there may exist (in fact, certainly do exist) other partitions of $C$ that would force this same set $X$ to be assigned measure $s / m$. What must be shown is that this happens if and only if $r m=s n$. This is done in Lemma 4 below.

The construction in the previous paragraph yields a collection $\mathscr{R}$ of sets $X$ to which we can assign a rational number $\mu^{*}(X)$ as measure. On the sets in $\mathscr{R}$, this measure faithfully represents the preference relation (Lemmas 5 and 6 ) and is finitely additive (Lemma 7). 
The measure $\mu^{*}$ is extended to the rest of the sets in the algebra on which the preference relation is defined by mimicking inner and outer Lebesgue measure. For example, the outer measure of a set is defined to be the infimum of the measures of all sets in $\mathscr{R}$ which are preferred to the given set. We show that the inner and outer measures coincide (Lemma 9), that the resulting measure is finitely additive (Lemmas 15 and 16), and that it does, indeed, represent the preference relation with which we started (Lemmas 12 and 18).

Before getting to the details of the proof of Theorem 1, we introduce some terminology. If $R$ is a CD preference relation and $\mathscr{X}$ is an equipartition of some set $X$, then we let $|\mathscr{X}|$ denote the number of sets in $\mathscr{X}$, and we write "Y $\in[\mathscr{X}]^{r "}$ to indicate that $\mathscr{Y}$ is a subcollection of $\mathscr{X}$ of size $r$.

In the lemmas that follow, we will say that $X$ is preferred to $Y$ if $X R Y$ holds, that $X$ is strictly preferred to $Y$ if $X P Y$ holds, and that $X$ is equivalent to $Y$, denoted $X \equiv Y$, if $X R Y$ and $Y R X$.

Lemma 1. (i) (Monotonicity) If $X \supset Y$, then $X R Y$.

(ii) If $\mathscr{X}$ is an equipartition of $X$ and $\mathscr{Y}, \mathscr{Y}^{\prime} \in[\mathscr{X}]^{r}$, then $\bigcup \mathscr{Y} \equiv \bigcup \mathscr{Y}^{\prime}$.

(iii) If $X P \varnothing$ and $\mathscr{X}$ is an equipartition of $X$, then $Y P \varnothing$ for every $Y \in \mathscr{X}$.

(iv) If $X \equiv Y$, then $C-X \equiv C-Y$.

Proof. (i) Assume, for contradiction, that we have $X \supset Y$ and $Y P X$. Then, by WA, we have $[Y \cup(X-Y)] P[X \cup(X-Y)]$ since $Y \cap(X-Y)=\varnothing$. Hence, $X P X$, contradiction. Assertions (ii), (iii), and (iv) follow from WA in a similar fashion.

Lemma 2. If $\mathscr{X}$ is an equipartition of $X$ and $r, s \leq|\mathscr{X}|$, then the union of $r$ sets from $\mathscr{X}$ is strictly preferred to the union of $s$ sets from $\mathscr{X}$ iff $X P \varnothing$ and $r>s$.

Proof. Notice first that by Lemma 1(ii) the quantifiers in Lemma 2 can be taken to be either existential or universal. The right-to-left direction is an immediate consequence of Lemma 1 (iii) and WA. In the left-to-right direction, notice that $X P \varnothing$ follows from monotonicity. Moreover, $r>s$ follows because $s \geq r$ would contradict monotonicity and Lemma 1(ii).

Lemma 3. For any $X \in \mathscr{A}$ and natural numbers $n$ and $r$, the following are equivalent:

(1) There exists an equipartition $\mathscr{X}$ of $C$ of size $n$, and there exists a collection $\mathscr{Y} \in[\mathscr{X}]^{r}$ such that $X=\bigcup \mathscr{Y}$.

(2) There exists an equipartition $\mathscr{X}$ of $C$ of size $n$, and there exists a collection $\mathscr{Y} \in[\mathscr{X}]^{r}$ such that $X \equiv \bigcup \mathscr{Y}$.

(3) For every equipartition $\mathscr{X}$ of $C$ of size $n$ and every collection $\mathscr{Y} \in[\mathscr{X}]^{r}$, we have $X \equiv \bigcup \mathscr{Y}$.

Proof. (1) $\rightarrow(2)$ : Trivial

$(2) \rightarrow(3):$ Suppose $\mathscr{X}$ is an equipartition of $C$ of size $n$, and suppose $\mathscr{Y} \in[\mathscr{X}]^{r}$ and $X \equiv \cup \mathscr{Y}$. Suppose $\mathscr{X}^{\prime}$ is another equipartition of $C$ of size $n$, and suppose $\mathscr{Y}^{\prime} \in\left[\mathscr{X}^{\prime}\right]^{r}$. If some set in $\mathscr{X}$ were strictly preferred to some set in $\mathscr{X}^{\prime}$, then transitivity would imply that every set $\mathscr{X}$ is strictly preferred to every set in $\mathscr{X}^{\prime}$. It would then follow from WA that $C P C$, which is not true. Similarly, no set in $\mathscr{X}^{\prime}$ can be strictly preferred to any set in $\mathscr{X}$. Thus, 
every set in $\mathscr{Y}$ is equivalent to every set in $\mathscr{Y}^{\prime}$, so, since $|\mathscr{Y}|=\left|\mathscr{Y}^{\prime}\right|$, we have $\bigcup \mathscr{Y} \equiv \bigcup \mathscr{Y}^{\prime}$ by WA. Thus $X \equiv \bigcup \mathscr{Y}^{\prime}$, as desired.

$(3) \rightarrow(1):$ By PP, there exists an equipartition $\mathscr{X}$ of $C$ of size $n$. Choose $\mathscr{Y} \in[\mathscr{X}]^{r}$. By (3), we have $X \equiv \bigcup \mathscr{Y}$, and by Lemma 1 (iv) $C-X \equiv$ $U(\mathscr{X}-\mathscr{Y})$. Now let $\mathscr{Z}$ be an equipartition of $X$ of size $r$, and let $\mathscr{W}$ be an equipartition of $C-X$ of size $n-r$. Notice that if $A \in \mathscr{Z}, B \in \mathscr{Y}$, $E \in \mathscr{X}-\mathscr{Y}$, and $F \in \mathscr{W}$, then $A \equiv B$ and $E \equiv F$ by WA. But $B$ and $E$ come from the same equipartition. Thus, $B \equiv E$, so, by transitivity, $A \equiv F$. This implies that $\mathscr{Z} \cup \mathscr{W}$ is also an equipartition of $C$ of size $n$, $\mathscr{Z} \in[\mathscr{Z} \cup \mathscr{W}]^{r}$, and $X=\bigcup \mathscr{Z}$.

If any (and hence all) of the conditions in Lemma 3 hold, we will say that $(r, n)$ is good for $X$. It will turn out that $(r, n)$ is good for $X$ iff the measure of $X$ is $r / n$.

Lemma 4. Both $(r, n)$ and $(s, m)$ are good for some set $X$ iff $r m=s n$.

Proof. Suppose first that $(r, n)$ and $(s, m)$ are good for some set $X$ and $X$ is not strictly preferred to the empty set. Then both $r$ and $s$ must be zero, so $r m=s n$, as desired. If $(r, n)$ and $(s, m)$ are good for $X$ and $X$ is strictly preferred to the empty set, then we let $\mathscr{X}$ be an equipartition of $C$ of size $m n$. By WA, a clumping together of blocks of size $n$ from $\mathscr{X}$ yields an equipartition of $C$ of size $m$, and a clumping together of blocks of size $m$ from $\mathscr{X}$ yields an equipartition of $C$ of size $n$. Thus, $X$ is equivalent to the union of $r$ blocks of size $m$ and the union of $s$ blocks of size $n$. By Lemma 2, $r m=s n$, as desired.

For the converse, suppose $r m=s n$ and let $\mathscr{X}$ be any equipartition of $C$ of size $m n$. Let $X$ be the union of any $r m$ sets from $\mathscr{X}$. Then a clumping together blocks of size $m$ in the obvious way shows that $(r, n)$ is good for $X$, and a clumping together of blocks of size $n$ (again in the obvious way) shows that $(s, m)$ is good for $X$.

Let the collection $\mathscr{R} \subset \mathscr{A}$ be defined by:

$$
X \in \mathscr{R} \quad \text { iff }(r, n) \text { is good for } X \text { for some } r \text { and } n \text {. }
$$

Now set $\mu^{*}(X)=r / n$ whenever $X \in \mathscr{R}$ and $(r, n)$ is good for $X$. Lemma 4 ensures that $\mu^{*}$ is well defined. The next few lemmas show that $\mu^{*}$ is finitely additive and that, for sets $X, Y \in \mathscr{R}, X R Y$ holds iff $\mu^{*}(X) \geq \mu^{*}(Y)$.

Lemma 5. If $X, Y \in \mathscr{R}$ and $X R Y$, then $\mu^{*}(X) \geq \mu^{*}(Y)$.

Proof. By getting a common denominator, we can assume $\mu^{*}(X)=r / n$ and $\mu^{*}(Y)=s / n$. Let $\mathscr{Z}$ be an equipartition of $C$ of size $n$. Choose $\mathscr{X} \in[\mathscr{Z}]^{r}$ and $\mathscr{Y} \in[\mathscr{Z}]^{s}$. Then $X \equiv \bigcup \mathscr{X}$ and $Y \equiv \bigcup \mathscr{Y}$, so $(\bigcup \mathscr{X}) R(\bigcup \mathscr{Y})$. Thus $r \geq s$ by Lemma 2 , since $C P \varnothing$.

Lemma 6. If $X, Y \in \mathscr{R}$ and $\mu^{*}(X) \geq \mu^{*}(Y)$, then $X R Y$.

Proof. Assume again that $\mu^{*}(X)=r / n$ and $\mu^{*}(Y)=s / n$ and let $\mathscr{Z}$ be an equipartition of $C$ of size $n$. Choose $\mathscr{X} \in[\mathscr{Z}]^{r}$ and $\mathscr{Y} \in[\mathscr{Z}]^{s}$. Then $X=\bigcup \mathscr{X}, Y=\bigcup \mathscr{Y}$, and, since $C P \varnothing$ and $r \geq s$, we have, by Lemma 2 ,

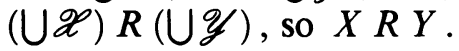

Lemma 7. If $X, Y \in \mathscr{R}$ and $X \cap Y=\varnothing$, then $\mu^{*}(X)+\mu^{*}(Y)=\mu^{*}(X \cup Y)$. Proof. Assume $\mu^{*}(X)=r / n$ and $\mu^{*}(Y)=s / n$, and let $\mathscr{Z}$ be an equipartition of $C$ of size $n$. If $r+s>n$, then choose $\mathscr{X} \in[\mathscr{Z}]^{r}$ and let $\mathscr{Y}=\mathscr{Z}-\mathscr{X}$. 
Then $X \equiv \bigcup \mathscr{X}$ and $Y P \cup \mathscr{Y}$ by Lemma 2 . Hence $(X \cup Y) P \cup \mathscr{Z}$ by WA. But $\cup \mathscr{Z}=C$, so this is impossible. Thus, $r+s \leq n$. Let $\mathscr{Y}^{\prime} \in[\mathscr{Z}]^{s}$ such that $\mathscr{X} \cap \mathscr{Y}^{\prime}=\varnothing$. Then $\mathscr{X} \cup \mathscr{Y}^{\prime}$ shows $(r+s, n)$ is good for $X \cup Y$, and hence, $\mu^{*}(X \cup Y)=(r+s) / n$.

Lemma 8. If $X, Y \in \mathscr{R}$ and $\mu^{*}(X)+\mu^{*}(Y) \leq 1$, then there exists $X^{\prime}, Y^{\prime} \in \mathscr{R}$ such that $X \equiv X^{\prime}, Y \equiv Y^{\prime}$, and $X^{\prime} \cap Y^{\prime}=\varnothing$.

Proof. Assume $\mu^{*}(X)=r / n$ and $\mu^{*}(Y)=s / n$. Then $r+s \leq n$. Let $\mathscr{Z}$ be an equipartition of $C$ of size $n$, and choose $\mathscr{X} \in[\mathscr{Z}]^{r}$ and $\mathscr{Y} \in[\mathscr{Z}]^{s}$ such that $\mathscr{X} \cap \mathscr{Y}=\varnothing$. Let $X^{\prime}=\bigcup \mathscr{X}$ and $Y^{\prime}=\bigcup \mathscr{Y}$.

In order to extend $\mu^{*}$ to a measure defined on all of $\mathscr{A}$, we define the following analogues of inner and outer Lebesgue measure: For $Y \in \mathscr{A}$, the inner measure of $Y$, denoted $\mu_{i}(Y)$, and the outer measure of $Y$, denoted $\mu_{o}(Y)$, are given by

$$
\mu_{i}(Y)=\sup \left\{\mu^{*}(X): Y R X\right\} \quad \text { and } \quad \mu_{o}(Y)=\inf \left\{\mu^{*}(X): X R Y\right\} .
$$

Lemma 9. For every $Y \in \mathscr{A}, \mu_{i}(Y)=\mu_{o}(Y)$.

Proof. Suppose first that we have $\mu_{i}(Y)<\mu_{o}(Y)$. Then we could choose a fraction $r / n$ so that

$$
\mu_{i}(Y)<r / n<\mu_{o}(Y) .
$$

Choose $X$ so that $\mu^{*}(X)=r / n$. Since $R$ is complete, either $Y R X$ or $X R Y$ holds. In the former case, we have $\mu_{i}(Y) \geq r / n$. In the latter case, we have $\mu_{o}(Y) \leq r / n$. Both are impossible.

Suppose now that we have $\mu_{o}(Y)<\mu_{i}(Y)$. Then we could choose $X^{\prime \prime}$ (from the collection of sets involved in the inf that gives us the outer measure of $Y$ ) and $X^{\prime}$ (from the collection of sets involved in the sup that gives us the inner measure of $Y$ ) so that $X^{\prime \prime} R Y$, and $Y R X^{\prime}$, and $\mu^{*}\left(X^{\prime \prime}\right)<\mu^{*}\left(X^{\prime}\right)$. But then we have $X^{\prime \prime} R X^{\prime}$, while Lemma 5 immediately implies that since $\mu^{*}\left(X^{\prime \prime}\right)<\mu^{*}\left(X^{\prime}\right)$, we have $X^{\prime} P X^{\prime \prime}$. This completes the proof of Lemma 9.

The desired measure $\mu$ on the algebra $\mathscr{A}$ is now obtained by setting $\mu(Y)$ to be the common value of the inner and outer measure of $Y$. It remains only to show that $\mu$ is finitely additive and that it induces the preference relation with which we started.

Lemma 10. If $X \cap Y=\varnothing, \mu(X)+\mu(Y)<1$, and $\varepsilon>0$, then there exists $X^{\prime}, Y^{\prime} \in \mathscr{R}$ such that

(i) $X^{\prime} \cap Y^{\prime}=\varnothing$,

(ii) $X^{\prime} R X$ and $Y^{\prime} R Y$, and

(iii) $\mu^{*}\left(X^{\prime}\right) \leq \mu(X)+\varepsilon$ and $\mu^{*}\left(Y^{\prime}\right) \leq \mu(Y)+\varepsilon$.

Proof. Without loss of generality, assume that $\mu(X)+\mu(Y)+2 \varepsilon \leq 1$. Since $\mu=\mu_{o}$, we can choose $X^{\prime \prime}, Y^{\prime \prime} \in \mathscr{R}$ so that

(i) $X^{\prime \prime} R X$ and $Y^{\prime \prime} R Y$, and

(ii) $\mu^{*}\left(X^{\prime \prime}\right) \leq \mu(X)+\varepsilon$ and $\mu^{*}\left(Y^{\prime \prime}\right) \leq \mu(Y)+\varepsilon$.

Notice that $\mu^{*}\left(X^{\prime \prime}\right)+\mu^{*}\left(Y^{\prime \prime}\right) \leq \mu(X)+\mu(Y)+2 \varepsilon \leq 1$, and so Lemma 8 guarantees the existence of $X^{\prime}, Y^{\prime} \in \mathscr{R}$ such that $X^{\prime} \equiv X^{\prime \prime}, Y^{\prime} \equiv Y^{\prime \prime}$, and $X^{\prime} \cap Y^{\prime}=\varnothing$. But then $\mu^{*}\left(X^{\prime}\right)=\mu^{*}\left(X^{\prime \prime}\right)$ and $\mu^{*}\left(Y^{\prime}\right)=\mu^{*}\left(Y^{\prime \prime}\right)$ by Lemma 5. Thus $X^{\prime}$ and $Y^{\prime}$ are as desired. 
Lemma 11. If $X \cap Y=\varnothing, \mu(X)+\mu(Y) \leq 1$, and $\varepsilon>0$, then there exist $X^{\prime}, Y^{\prime} \in \mathscr{R}$ such that

(i) $X^{\prime} \cap Y^{\prime}=\varnothing$,

(ii) $X R X^{\prime}$ and $Y R Y^{\prime}$, and

(iii) $\mu^{*}\left(X^{\prime}\right) \geq \mu(X)-\varepsilon$ and $\mu^{*}\left(Y^{\prime}\right) \geq \mu(Y)-\varepsilon$.

Proof. Since $\mu=\mu_{i}$, we can choose $X^{\prime \prime}, Y^{\prime \prime} \in \mathscr{R}$ so that

(i) $X R X^{\prime \prime}$ and $Y R Y^{\prime \prime}$, and

(ii) $\mu(X) \geq \mu^{*}\left(X^{\prime \prime}\right) \geq \mu(X)-\varepsilon$ and $\mu(Y) \geq \mu^{*}\left(Y^{\prime \prime}\right) \geq \mu(Y)-\varepsilon$.

Notice that $\mu^{*}\left(X^{\prime \prime}\right)+\mu^{*}\left(Y^{\prime \prime}\right)<\mu(X)+\mu(Y) \leq 1$ by assumption. Hence, Lemmas 8 and 5 yield the desired result as in the above lemma.

Lemma 12. If $X R Y$, then $\mu(X) \geq \mu(Y)$, and so, if $X \supset Y$, then $\mu(X) \geq$ $\mu(Y)$.

Proof. Immediate, since $X R Y$ implies the inner measure of $X$ is at least as large as the inner measure of $Y$. The last clause follows from this and monotonicity.

Lemma 13. For $X \in \mathscr{A}, \mu(X)+\mu(C-X)=1$.

Proof. We have

$$
\begin{aligned}
\mu(X) & =\mu_{i}(X)=\sup \left\{\mu^{*}(Z): X R Z\right\} \\
& =\sup \left\{\mu^{*}(Z):(C-Z) R(C-X)\right\}=\sup \left\{\mu^{*}(C-Z): Z R(C-X)\right\} \\
& =\sup \left\{1-\mu^{*}(Z): Z R(C-X)\right\}=1-\inf \left\{\mu^{*}(Z): Z R(C-X)\right\} \\
& =1-\mu_{0}(C-X)=1-\mu(C-X) .
\end{aligned}
$$

Thus, $\mu(X)+\mu(C-X)=1$.

Lemma 14. If $X \cap Y=\varnothing$, then $\mu(X)+\mu(Y) \leq 1$.

Proof. Since $X \cap Y=\varnothing$, we have $Y \subset C-X$. Thus, $\mu(Y) \leq \mu(C-X)$ by Lemma 12. It now follows from Lemma 13 that $\mu(X)+\mu(Y) \leq \mu(X)+$ $\mu(C-X)=1$.

Lemma 15. If $X \cap Y=\varnothing$, then $\mu(X \cup Y) \geq \mu(X)+\mu(Y)$.

Proof. Assume $X \cap Y=\varnothing$ and $\varepsilon>0$. By Lemmas 14 and 11, we can choose $X^{\prime}, Y^{\prime} \in \mathscr{R}$ such that

(i) $X^{\prime} \cap Y^{\prime}=\varnothing$,

(ii) $X R X^{\prime}$ and $Y R Y^{\prime}$, and

(iii) $\mu^{*}\left(X^{\prime}\right) \geq \mu(X)-\varepsilon / 2$ and $\mu^{*}\left(Y^{\prime}\right) \geq \mu(Y)-\varepsilon / 2$.

By (i) and Lemma 7, $\mu^{*}\left(X^{\prime} \cup Y^{\prime}\right)=\mu^{*}\left(X^{\prime}\right)+\mu^{*}\left(Y^{\prime}\right)$. By (ii) and WA, $(X \cup Y) R\left(X^{\prime} \cup Y^{\prime}\right)$. Thus, by Lemma 12, $\mu(X \cup Y) \geq \mu\left(X^{\prime} \cup Y^{\prime}\right)$. This yields

$$
\mu(X \cup Y) \geq \mu\left(X^{\prime} \cup Y^{\prime}\right)=\mu^{*}\left(X^{\prime} \cup Y^{\prime}\right)=\mu^{*}\left(X^{\prime}\right)+\mu^{*}\left(Y^{\prime}\right) \geq \mu(X)+\mu(Y)-\varepsilon
$$

Since this is true for every $\varepsilon>0$, we have $\mu(X \cup Y) \geq \mu(X)+\mu(Y)$, as desired.

Lemma 16. If $X \cap Y=\varnothing$, then $\mu(X \cup Y) \leq \mu(X)+\mu(Y)$.

Proof. Assume $X \cap Y=\varnothing$ and $\varepsilon>0$. By Lemma 14, $\mu(X)+\mu(Y) \leq 1$. If $\mu(X)+\mu(Y)=1$, the conclusion is trivial. Otherwise, Lemma 10 allows us to 
choose $X^{\prime}, Y^{\prime} \in \mathscr{R}$ such that

(i) $X^{\prime} \cap Y^{\prime}=\varnothing$,

(ii) $X^{\prime} R X$ and $Y^{\prime} R Y$, and

(iii) $\mu^{*}\left(X^{\prime}\right) \leq \mu(X)+\varepsilon / 2$ and $\mu^{*}\left(Y^{\prime}\right) \leq \mu(Y)+\varepsilon / 2$.

By (i) and Lemma 7, $\mu^{*}\left(X^{\prime} \cup Y^{\prime}\right)=\mu^{*}\left(X^{\prime}\right)+\mu^{*}\left(Y^{\prime}\right)$. By (i), (ii), and WA, $\left(X^{\prime} \cup Y^{\prime}\right) R(X \cup Y)$. Thus, by Lemma 12, $\mu\left(X^{\prime} \cup Y^{\prime}\right) \geq \mu(X \cup Y)$. This yields

$$
\begin{aligned}
\mu(X \cup Y) & \leq \mu\left(X^{\prime} \cup Y^{\prime}\right)=\mu^{*}\left(X^{\prime} \cup Y^{\prime}\right) \\
& =\mu^{*}\left(X^{\prime}\right)+\mu^{*}\left(Y^{\prime}\right) \leq \mu(X)+\mu(Y)+\varepsilon .
\end{aligned}
$$

Since this is true for every $\varepsilon>0$, we have $\mu(X \cup Y) \leq \mu(X)+\mu(Y)$, as desired.

Lemma 17. If $T P \varnothing$, then $\mu(T)>0$.

Proof. If $T P \varnothing$, then the Archimedean Postulate AP guarantees that $T R X$ for some $X$ with $\mu^{*}(X)=1 / n$ for sufficiently large $n$. Lemma 12 now guarantees that $\mu(T) \geq \mu(X)=\mu^{*}(X)>0$. Notice that this is the first time we have had to appeal to the Archimedean Postulate.

Lemma 18. If $\mu(X) \geq \mu(Y)$, then $X R Y$.

Proof. Assume $Y P X$. By the Trimming Postulate TP, we can choose $T \subset Y$ so that $Y-T \equiv X$, and, thus, by Lemma 12, $\mu(Y-T)=\mu(X)$. By WA, $T P \varnothing$, so, by Lemma $17, \mu(T)>0$. Lemmas 15 and 16 now yield that $\mu(Y)=\mu(Y-T)+\mu(T)=\mu(X)+\mu(T)>\mu(X)$, as desired.

Notice that a consequence of the fact that we needed the Archimedean Postulate only for the proof of Lemmas 17 and 18 is that we immediately obtain the following:

Corollary 1. If $R$ is a CD preference relation on $\mathscr{A}$, then (even if $R$ is not Archimedean) there exists a finitely additive measure $\mu$ on $\mathscr{A}$ such that if $A R B$, then $\mu(A) \geq \mu(B)$. Thus, if we have $A \equiv B$, then $\mu(A)=\mu(B)$.

As pointed out by the referee, mathematical economists refer to a function $u: \mathscr{A} \rightarrow \mathbb{R}$ as a utility for a preference relation $R$ if for all $X, Y \in \mathscr{A}$ we have $u(X) \geq u(Y)$ whenever $X R Y$, and a utility representation for $R$ if we have $u(X) \geq u(Y)$ precisely when $X R Y$. Thus, Theorem 1 asserts the existence of an additive utility representation for an Archimedean $C D$ relation, while Corollary 1 asserts the existence of an additive utility for even a nonArchimedean $\mathrm{CD}$ relation.

One should note, however, that if the CD preference relation $R$ in the Corollary is not Archimedean, then the measure $\mu$ obtained in the proof of the theorem will be such that for some pair of sets $A$ and $B$ one has $A P B$ even though $\mu(A)=\mu(B)$. Intuitively, one can think of $A$ as being preferred "infinitesimally" to $B$. Notice that any set of measure zero that is strictly preferred to the empty set must be an infinitesimal.

In [BT], a constructive procedure is given for obtaining an envy-free allocation of a cake $C$ among $n$ players when each player's preferences are given by a finitely additive measure defined on some algebra $\mathscr{A}$ of subsets of $C$. The question of whether or not such a procedure exists (for $n>3$ ) in the case where preferences are given by CD preference relations is open. The above corollary, however, allows us to obtain the following partial result in the positive direction. 
Corollary 2. Given $n$ players whose preferences over subsets of $C$ are given by $C D$ preference relations, there exists an ordered partition $\left\{X_{1}, \ldots, X_{n}\right\}$ of $C$ that is "infinitesimally close to being envy-free". That is, for each $i$ there is a set $S_{i}$ that Player $i$ thinks is an infinitesimal, and he or she regards $\left\{X_{1}-S_{i}, \ldots, X_{n}-S_{i}\right\}$ as an envy-free allocation of $C-S_{i}$.

Proof. Given CD preference relations $R_{1}, \ldots, R_{n}$ on some algebra $\mathscr{A}$ of subsets of $C$, let $\mu_{1}, \ldots, \mu_{n}$ be the measures guaranteed to exist by Corollary 1. Now apply the envy-free procedure from [BT] to obtain the ordered partition $\left\{X_{1}, \ldots, X_{n}\right\}$ with $\mu_{i}\left(X_{i}\right) \geq \mu_{i}\left(X_{j}\right)$ for every $i, j$. Assume that Player $i$ experiences some envy. Then we have $X_{j} P_{i} X_{i}$ for some $j$. Proceeding as in the proof of Lemma 18, we can use the Trimming Postulate TP to get $T_{j} \subset X_{j}$ so that $X_{j}-T_{j} \equiv_{i} X_{i}$. Thus, $\mu_{i}\left(X_{j}-T_{j}\right)=\mu_{i}\left(X_{i}\right) \geq \mu_{j}\left(X_{j}\right)$. Thus, $\mu_{i}\left(T_{j}\right)=0$, so, since $T_{j}$ is strictly preferred to the empty set (by WA), we have that $T_{j}$ is an infinitesimal (according to Player $i$ ). It is easy to see that a finite union of infinitesimals is again an infinitesimal. Thus, we can let $S_{i}$ be the union of all the sets $T_{j}$.

\section{Proof OF THEOREM 2}

Our construction of a $C D$ preference relation that does not satisfy the Archimedean Postulate will proceed by amalgamating preferences in a way that ultrafilter-theorists will find familiar. The "cake" will be a subset of the plane (which could be taken to be compact by trivial modifications of what we are about to do).

For each $n \in N$, let $C_{n}$ be the open disk of radius $1 / 2$ centered at the point $(n, 0)$ on the $x$-axis, and let $C$ be the union of this infinite collection of disks. Let $\mu$ denote Lebesgue measure in the plane, and let $\mathscr{A}$ denote the algebra of Borel subsets of $C$. Let $\mathscr{U}$. be any nonprinciple ultrafilter on $N$, and for any subset $X$ of $C$, let $X_{n}$ denote $X \cap C_{n}$.

We can now define the desired preference relation for pairs of sets from the algebra $\mathscr{A}$ as follows: if $X, Y \in \mathscr{A}$, set

$$
X R Y \quad \text { iff } \quad\left\{n \in N: \mu\left(X_{n}\right) \geq \mu\left(Y_{n}\right)\right\} \in \mathscr{U} .
$$

Notice that

$$
X \equiv Y \quad \text { iff } \quad\left\{n \in N: \mu\left(X_{n}\right)=\mu\left(Y_{n}\right)\right\} \in \mathscr{U}
$$

and

$$
X P Y \quad \text { iff } \quad\left\{n \in N: \mu\left(X_{n}\right)>\mu\left(Y_{n}\right)\right\} \in \mathscr{U} .
$$

It is trivial to check that $R$ is reflexive, transitive, and complete. The Partitioning Postulate holds, since for any $X \in \mathscr{A}$ and any $k \in N$ we can partition each "piece" $X_{n}$ of $X$ into $k$ sets of equal Lebesgue measure. The verifications of the Trimming Postulate and the Weak Additivity Postulate are just as easy.

To see that the Archimedean Postulate fails, choose, for each $n \in N$, a set $X_{n} \subset C_{n}$ of Lebesgue measure $1 / n$. Let $X$ be the union of the $X_{n}$ 's. Then $X$ is strictly preferred to the empty set. However, if we partition $C$ into $k$ equivalent sets, then, for all $n$ in a set in $\mathscr{U}$, we have that each of the $k$ sets intersects $C_{n}$ in a set of Lebesgue measure $1 / k$. Since $1 / k$ is larger than the 
Lebesgue measure of $X_{n}$ for all but finitely many such $n$, we have that each of the $k$ sets is strictly preferred to $X$. This completes the proof of Theorem 2 .

\section{ACKNOWLEDGMENT}

We thank the referee for several helpful suggestions and for bringing to our attention some related work done independently, and much earlier, by Debreu [D], Koopman [K], Peleg [P], Savage [S], and Villegas [V]. Because their contexts and assumptions differ from ours, we have chosen to make the present paper self-contained.

\section{REFERENCES}

[BT] S. J. Brams and A. D. Taylor, An envy-free cake division protocol, Amer. Math. Monthly 102 (1995), 9-18.

[D] G. Debreu, Continuity properties of Paretian utility, Internat. Econom. Rev. 5 (1964), 285-293.

[DS] L. E. Dubins and E. H. Spanier, How to cut a cake fairly, Amer. Math. Monthly 68 (1961), $1-17$.

[G] D. Gale, Mathematical entertainments, Math. Intelligencer 15 (1993), 48-52.

[K] B. O. Koopman, The axioms and algebra of intuitive probability, Ann. of Math. (2) 41 (1940), 269-292.

[P] B. Peleg, Utility functions for partially ordered topological spaces, Econometrica 38 (1970), 93-96.

[S] L. J. Savage, The foundations of statistics, Dover, New York, 1972.

[V] C. Villegas, On qualitative probability $\sigma$-algebras, Ann. of Math. Statist. 35 (1964), 17871796.

Department of Mathematics, Union College, Schenectady, New York 12308 